\title{
Prevención de eventos tromboembólicos con aspirina exclusiva post reemplazo valvular aórtico con bioprótesis
}

\author{
Enrique Seguel ${ }^{1,2}$, Roberto González $z^{1,2}$, Aleck Stockins ${ }^{1,2}$ \\ Emilio Alarcón ${ }^{1,2}$, Nicolás Silva ${ }^{3}$, Gonzalo Concha ${ }^{3}$
}

\footnotetext{
1.- Departamento de Cirugía, Facultad de Medicina, Universidad de Concepción; 2.- Centro Cardiovascular, Hospital Dr. Guillermo Grant Benavente de Concepción. 3.- Alumno de medicina
}

Introducción: La principal ventaja de las bioprótesis es no requerir tratamiento anticoagulante. Sin embargo, algunas guías de manejo clínico recomiendan este tratamiento los primeros meses post cirugía. En los últimos años varios autores han demostrado la seguridad del uso exclusivo de aspirina en los primeros 3 meses después del reemplazo valvular aórtico con bioprótesis. Objetivo: Evaluar la morbimortalidad y complicaciones trombo embólicas y hemorrágicas en pacientes sometidos a reemplazo valvular aórtico (RVA) con bioprótesis tratados exclusivamente con aspirina (100 mg) los primeros tres meses post cirugía.

Métodos: Estudio retrospectivo de 229 pacientes (137 hombres. edad 65,3 $\pm 11,76$ años) operados de RVA con bioprótesis entre junio 2006 y diciembre 2011. Hubo 178 cirugías aisladas y 51 combinadas y 20 pacientes te- nían endocarditis. Se estudió la morbimortalidad, complicaciones trombo embólicas y sangrado a 30 y 90 días y en el seguimento alejado hasta el 30 de junio de 2012. Resultados: A 30 días hubo 4 accidentes cerebrovasculares, 3 accidentes isquémicos transitorios y una isquemia mesentérica. Fallecieron 8 pacientes $(3,5 \%)$. A los 90 días hubo 2 hemorragias (1 hemorragia digestiva, 1 hemotórax), no hubo nuevos eventos trombo embólicos ni otros fallecidos. El seguimiento promedio fue $27.8 \pm 17,7$ meses (rango $6-72$ meses). Durante el seguimiento fallecieron 17 pacientes y no se registraron eventos trombo embólicos ni hemorrágicos.

Conclusión: En pacientes operados de reemplazo valvular aórtico con bioprótesis el uso exclusivo de aspirina fue seguro para prevenir complicaciones trombo embólicas.

\section{Correspondencia:}

Dr. Enrique Seguel Soto

Departamento de Cirugía, Facultad de Medicina, Universidad de Concepción.

enseguel@udec.cl 


\section{Prevention of thrombo-embolic events with aspirin alone after aortic valve replacement with a biologic prosthesis}

Background: The abscense of a need for anticoagulant therapy is a significant advantage of biologic valve prosthesis. However, according to some clinical guidelines conventional anti-coagulant therapy is recommended for the initial 3 months following aortic valve replacement.

Aim: The aim of this study was to evaluate morbimortality and thrombo-embolic events in patients undergoing aortic valve replacement with a bioprosthesis receiving aspirin during the first 3 months after surgery. Methods: Data on 229 patients (137 males), aged $65.3 \pm 11.8$ years who received biologic aortic valve prosthesis between June 2006 and December 2011 was retrospectively analyzed. 51 patients underwent combined (coronary and/or mitral valve surgery) and 20 patients had infectious endocarditis. Morbidity, mortality, thrombo-embolic and hemorrhagic events were tabulated up to June 30, 2012.

Results: During the first 30 days after surgery there were 4 cerebro-vascular events and 1 episode of mesenteric ischemia. Operative (30 day) mortality was $3.5 \%$ (8 patients). At 90 days, 2 patients had a hemorrhagic event (GI bleeding and hemothorax, respectively), but no further embolic events or deaths occurred. Patients were followed for a mean of $27.8 \pm 17,7$ months (range 6 to 72 months). 17 patients died but no cases of embolism or bleeding were observed.

Conclusion: Aspirin was safe and effective for prevention of thrombo-embolic complications following aortic valve replacement with a biologic prosthesis

Keywords: aortic valve replacement, biologic valve prosthesis, anticoagulant treatment, aspirin.
Introducción: Durante los últimos años hemos observado un progresivo aumento de la patología valvular aórtica, probablemente a causa del envejecimiento de la población y a un mejor acceso al diagnóstico y atención de salud ${ }^{1}$.

El tratamiento estándar de esta patología es el reemplazo de la válvula enferma por vía quirúrgica con una prótesis, cirugía que se ha convertido en una de las intervenciones cardíacas más frecuentemente realizadas hoy en día. De acuerdo a los registros de la base de datos de la Asociación Europea de Cirugía Cardiotorácica, de las 404.721 cirugías cardíacas registradas entre los años 2006 y 2008, $69.902(17 \%)$ correspondieron a reemplazos valvulares aórticos aislados o asociados a cirugía coronaria ${ }^{2}$.

Para la cirugía de reemplazo valvular existen varias alternativas de prótesis: Las prótesis mecánicas (de bola, de disco o de dos aletas), que tienen en común una baja tasa de deterioro estructural y una duración teóricamente 
“ilimitada". Por esta razón son recomendadas a pacientes jóvenes o con una expectativa de vida larga. Estas prótesis se asocian a la formación de trombos que pueden embolizar u obstruir el mecanismo protésico y requieren del uso de un tratamiento anticoagulante oral (TACO) de por vida para prevenirlos ${ }^{3}$.

El tratamiento anticoagulante requiere de una buena adherencia del paciente y de un control estricto para disminuir sus complicaciones. Cannegieter y cols, publicaron en 1995 una serie de 1.608 pacientes con un seguimiento equivalente a 6472 pacientes-año. El tratamiento anticoagulante se mantuvo en rangos efectivos sólo en el $61 \%$ de ellos y la incidencia de un primer evento tanto trombo embólico como hemorrágico fue de un 3,5\% por año ${ }^{4}$. En nuestro medio es probable que la tasa de adherencia sea menor y las tasas de complicaciones más altas.

Una alternativa a las prótesis mecánicas son las biopótesis. Estas corresponden a xenoinjertos elaborados con tejido animal. Existen modelos que utilizan la válvula aórtica de un cerdo (porcinas) montada, o no, en un soporte y otras manufacturadas del pericardio bovino. Con el tiempo estas prótesis tienden a sufrir falla estructural y pueden requerir una re-intervención para su reemplazo. $\mathrm{Su}$ gran ventaja radica en que no requieren de un tratamiento anticoagulante a permanencia. Están recomendadas en los pacientes mayores de 60 años, pacientes en los cuales la anticoagulación puede ser peligrosa (mujeres en edad fértil, pacientes que desarrollan actividades de riesgo, contraindicaciones de la anticoagulación, etc.) o pacientes con expectativa de vida corta. ${ }^{5}$

A pesar de no requerir anticoagulación de largo plazo, las antiguas recomendaciones internacionales y los fabricantes de estas prótesis sugerían su uso los primeros tres meses luego de la cirugía de reemplazo valvular aórtico ${ }^{6-10}$. Este periodo, si bien breve, obliga a usar TACO en pacientes en los que este tratamiento se desea evitar.

Varios autores y centros dejaron de utilizar anticoagulación formal y comenzaron a utilizar aspirina durante el periodo post operatorio, observando que no existía una mayor tasa de complicaciones ${ }^{11}$ - 15 .

Nuestro equipo comenzó a utilizar bioprótesis el año 2006. Basados en estos reportes y en las ventajas de evitar la anticoagulación de nuestros pacientes, decidimos utilizar aspirina en forma exclusiva durante el post operatorio en los pacientes que no tenían otra indicación de uso de TACO.
El objetivo de este trabajo fue evaluar las complicaciones trombo - embólicas en pacientes operados de reemplazo valvular aórtico con biopótesis tratados con aspirina exclusiva durante los primeros tres meses después de la cirugía.

Método: Pacientes: Retrospectivamente, se analizaron 229 pacientes operados de reemplazo valvular aórtico con bioprótesis en el Hospital "Dr. Guillermo Grant Benavente" de Concepción entre mayo 2006 y diciembre 2011. Se excluyeron los pacientes con datos incompletos ( $\mathrm{N}=22$ ) y aquellos con indicación de TACO por cualquier causa $(\mathrm{N}=35)$. Los datos fueron obtenidos de las fichas clínicas y de la base de datos informática del hospital.

Hubo 137 hombres y 92 mujeres. La edad promedio fue $65,3 \pm 11,7$ años (rango 15 - 95 años). Hubo 52 pacientes $(22,7 \%)$ menores de 60 años.

Entre las patologías asociadas destacan la hipertensión arterial en 164 pacientes $(71,6 \%)$; tabaquismo en 62 (27\%); diabetes mellitus en $60(26,2 \%)$; enfermedad pulmonar obstructiva crónica en $17(7,4 \%)$; insuficiencia renal en $13(5,7 \%)$, de los cuales 4 se encontraban en diálisis; y 8 pacientes $(3,5 \%)$ con antecedente de un accidente vascular encefálico. Hubo 7 pacientes con cirugía cardíaca previa (3\%). Todos los pacientes se estudiaron con ecocardiograma de superficie antes de su cirugía y 20 pacientes presentaban una endocarditis activa.

Los antecedentes clínicos de resumen en la Tabla 1.

\begin{tabular}{|l|c|c|}
\hline \multicolumn{2}{|l|}{ Tabla 1. Características clínicas de los pacientes. } \\
\hline $\mathrm{N}$ & 229 & \\
\hline Hombres & 137 & $59,8 \%$ \\
\hline Mujeres & 92 & $40,2 \%$ \\
\hline Edad & $65,3 \pm 11,7$ años & (rango $15-95$ años) \\
\hline Menores de 60 años & 52 & $22,7 \%$ \\
\hline Patologías asociadas & & \\
\hline Hipertensión arterial & 164 & $71,6 \%$ \\
\hline Tabaquismo & 62 & $27 \%$ \\
\hline Diabetes mellitus & 60 & $26,2 \%$ \\
\hline Enfermedad Pulmonar & 17 & $7,4 \%$ \\
\hline Insuficiencia Renal Crónica & 13 & $5,7 \%$ \\
\hline Diálisis & 4 & $1,7 \%$ \\
\hline Accidente Cerebro Vascular previo & 8 & $3,5 \%$ \\
\hline Cirugía Previa & 7 & $3 \%$ \\
\hline Endocarditis Activa & 20 & $8,7 \%$ \\
\hline
\end{tabular}

Clínicamente, 85 pacientes $(37,1 \%)$ se encontraban en capacidad funcional I - II y $144(62,9 \%)$ en capacidad funcional III - IV. 
La patología valvular correspondió a estenosis pura en 153 pacientes $(66,8 \%)$, insuficiencia en 43 pacientes $(18,8 \%)$ y doble lesión en 33 pacientes $(14,4 \%)$. El estudio mostró patología mitral asociada en 44 pacientes (43 con insuficiencia y 1 con estenosis). Insuficiencia tricuspídea presentaron 14 pacientes.

La fracción de eyección promedio del ventrículo izquierdo fue $56 \pm 12,7 \%$ (rango $15-85 \%$ ). Correspondió a 183 pacientes $(79,9 \%)$ con función normal ( $\mathrm{FE}>50 \%) ; 39$ pacientes ( $17 \%)$ con disfunción moderada $(30-50 \%)$ y 7 pacientes (3\%) con disfunción grave ( $\mathrm{FE}<30 \%)$.

Se realizó estudio coronario a los pacientes mayores de 50 años. Se encontró enfermedad coronaria significativa en 38 pacientes $(16,6 \%)$ y correspondieron a 14 pacientes con lesión de un vaso, 9 pacientes con lesión de dos y 15 pacientes con lesión de tres vasos (Tabla 2).

\begin{tabular}{|c|c|c|}
\hline \multicolumn{3}{|l|}{ CAPACIDAD FUNCIONAL NYHA } \\
\hline I- II & 85 & $37,1 \%$ \\
\hline III - IV & 114 & $62,9 \%$ \\
\hline \multicolumn{3}{|l|}{ Patología valvular } \\
\hline Estenosis & 153 & $66,8 \%$ \\
\hline Insuficiencia & 43 & $18,8 \%$ \\
\hline Doble Lesión & 33 & $14,4 \%$ \\
\hline Enfermedad mitral asociada & 44 & $19,2 \%$ \\
\hline Insuficiencia tricuspídea & 14 & $6,1 \%$ \\
\hline $\begin{array}{l}\text { Fracción de eyección } \\
\text { del ventrículo izquierdo }\end{array}$ & $56 \pm 12,7 \%$ & (rango $15-85 \%$ ) \\
\hline$>50 \%$ & 183 & $79,9 \%$ \\
\hline $30-50 \%$ & 39 & $17 \%$ \\
\hline$<30 \%$ & 7 & $3 \%$ \\
\hline Enfermedad coronaria & 38 & $16,6 \%$ \\
\hline Un vaso & 14 & \\
\hline Dos Vasos & 9 & \\
\hline Tres Vasos & 15 & \\
\hline
\end{tabular}

Intervenciones: Todas las intervenciones se realizaron por esternotomía media con anestesia general, anticoagulación plena con heparina y circulación extracorpórea. Una vez pinzada la aorta se procedió a la protección del corazón mediante infusión de solución de cardiolpegia sanguínea por vía anterógrada y/o retrógrada. La válvula aórtica se abordó por aortotomía transversa. Luego de la resección de la válvula enferma se procedió al implante de las bioprótesis en posición supra anular fijadas con suturas separadas de poliester 2-0 (Ethibond Excel ${ }^{\circledR}$ ) apoyadas en pledgets de teflón.

Se utilizaron cuatro modelos de biopótesis. Tres de ellas corresponden a prótesis porcinas: Biocor ${ }^{\circledR}$ (St Jude Medical Inc, St. Paul, Minn.) en 160 pacientes (69,9\%); Car- pentier Edwards ${ }^{\circledR}$ (Edwards Lifesciences, Irvine, CA) en 61 pacientes $(26,6 \%)$ y Hancock II ${ }^{\circledR}$ (Medtronic Corp., Minneapolis, MN) en 3 pacientes (1,3\%). Se utilizó la prótesis de pericardio bovino Perimount ${ }^{\circledR}$ (Edwards Lifesciences, Irvine, $C A)$ en 5 pacientes $(2,2 \%)$.

La mediana del tamaño de las prótesis utilizadas fue de $23 \mathrm{~mm}$ (rango 19-27 mm).

Se realizaron 182 reemplazos valvulares aórticos aislados y 47 cirugías combinadas (34 cirugías coronarias, 6 reemplazos valvulares mitrales, 1 reemplazo tricuspídeo y 6 reemplazos de aorta ascendente). Tabla 3 .

\begin{tabular}{|c|c|c|}
\hline \multicolumn{3}{|l|}{ PRÓTESIS } \\
\hline Biocor ${ }^{\circledR}$ & 160 & $69,9 \%$ \\
\hline Carpentier Edwards $®$ & 61 & $26,6 \%$ \\
\hline Hancok II ${ }^{\circ}$ & 3 & $1,3 \%$ \\
\hline Perimount $₫$ & 5 & $2,2 \%$ \\
\hline Tamaño (mediana) & 23 & \\
\hline Reemplazo aórtico aislado & 182 & $79,5 \%$ \\
\hline Cirugías combinadas & 47 & $20,5 \%$ \\
\hline Cirugía Coronaria & 34 & \\
\hline Reemplazo mitral & 6 & \\
\hline Reemplazo tricuspídeo & 1 & \\
\hline Reemplazo aorta ascendente & 6 & \\
\hline
\end{tabular}

Los pacientes recibieron aspirina en dosis de $100 \mathrm{mg}$ vía oral desde el primer día post operatorio. El tratamiento se mantuvo por los primeros tres meses.

Eventos: se tabulan las complicaciones y la mortalidad operatoria hasta 30 días o al alta del paciente si la hospitalización fue mayor a 30 días. Se tabulan los eventos trombo embólicos, sangrado y mortalidad hasta 90 días. Se consideró como eventos trombo embólicos todo accidente vascular encefálico isquémico clínico y confirmado con tomografía computada. Además, cualquier evento de embolia periférica clínica. Se consideró como evento hemorrágico cualquier accidente vascular encefálico hemorrágico y cualquier evento de sangrado interno (digestivo, ginecológico, etc.), o externo evidenciado clínicamente.

Seguimiento: Se realizó seguimiento de los pacientes que sobrevivieron a la cirugía hasta el 31 de junio de 2012. La sobrevida alejada se evaluó con los datos del Registro Civil e Identificación. Los eventos trombo embólicos y sangrado se evaluaron de acuerdo a los registros de las fichas clínicas y la base de datos del hospital. 
Resultados: Morbimortalidad Operatoria: Se registraron 9 complicaciones quirúrgicas que correspondieron a 8 reoperaciones por sangrado perioperatorio y una por mediastinitis.

Se presentó arritmia completas por fibrilación auricular (AC x FA) en 28 pacientes (12,2\%), en todos los casos con menos de 24 horas de duración y que convirtieron a ritmo sinusal con terapia farmacológica. Cuatro pacientes $(1,7 \%)$ sufrieron un bloqueo aurículo - ventricular (BAV) transitorio y uno sufrió un $\mathrm{BAV}$ permanente que requirió el implante de un marcapasos $(0,4 \%)$.

Entre las complicaciones médicas hubo 8 neumonías $(3,5 \%)$ y 13 pacientes $(5,7 \%)$ sufrieron una insuficiencia renal aguda (uno de ellos requirió diálisis).

Se registraron 3 accidentes isquémicos transitorios (TIA) y cuatro accidentes vasculares encefálicos (AVE). Un paciente sufrió una isquemia mesentérica. Estas 8 complicaciones $(3,5 \%)$ se consideraron de origen embólico.

\begin{tabular}{|c|c|c|}
\hline \multicolumn{3}{|l|}{ COMPLICACIONES QUIRÚRGICAS } \\
\hline Reoperación por sangrado & 8 & $3,5 \%$ \\
\hline Mediastinitis & 1 & $0,4 \%$ \\
\hline \multicolumn{3}{|l|}{ COMPLICACIONES MÉDICAS } \\
\hline $\mathrm{AC} \times \mathrm{FA}$ & 28 & $12,2 \%$ \\
\hline Bloqueo Aurículo - Ventricular & 4 & $1,7 \%$ \\
\hline Marcapasos Definitivo & 1 & $0,4 \%$ \\
\hline Neumonía & 8 & $3,5 \%$ \\
\hline Insuficiencia Renal Aguda & 13 & $5,7 \%$ \\
\hline Diálisis & 1 & \\
\hline COMPLICACIONES EMBÓLICAS & 8 & $3,5 \%$ \\
\hline Accidente Isquémico Transitorio & 3 & $1,3 \%$ \\
\hline Accidente Cerebro Vascular & 4 & $1,7 \%$ \\
\hline Isquemia Mesentérica & 1 & $0,4 \%$ \\
\hline \multicolumn{3}{|l|}{ FALLECIDOS } \\
\hline Mortalidad Global & 8 & $3,5 \%$ \\
\hline Reemplazo Valvular Aislado & 4 & $2,2 \%$ \\
\hline
\end{tabular}

Fallecieron 8 pacientes (3,5\%): Tres por síndrome post cardiotomía y shock cardiogénico refractario; dos pacientes sufrieron un paro cardiorespiratorio (uno por fibrilación ventricular y otro por muerte súbita); un paciente falleció por sangrado en el post operatorio; un paciente con endocarditis falleció de un shock séptico y uno por isquemia mesentérica. Sólo este último fallecimiento se atribuyó a causa embólica $(0,4 \%)$.

Seguimiento a 90 días: un paciente reingresó al mes de su cirugía por una hemorragia digestiva alta. La endoscopía mostró erosiones gástricas. Un segundo paciente rein-

\begin{tabular}{|l|c|c|}
\hline \multicolumn{2}{|l|}{ Tabla 5: Seguimiento } \\
\hline Sobrevida & $28,8 \pm 17,7$ meses & (rango $1-72$ meses) \\
\hline Fallecidos & 5,3 años / 100 pacientes & \\
\hline Causa Cardiovascular & 17 & $7,7 \%$ \\
\hline Otras causas & 8 & $3,6 \%$ \\
\hline Seguimiento Clínico & $7,48 \pm 2,1$ meses & (rango $1-64$ meses) \\
\hline Eventos trombo embólicos & 1,2 años $/ 100$ pacientes & \\
\hline
\end{tabular}

gresó por un derrame pleural y se practicó un drenaje que dio salida a sangre antigua (hemotórax). En este período no falleció ningún paciente y no se registran eventos tromboembólicos.

Seguimiento alejado: Se realizó a través de los datos del Servicio de Registro Civil e Identificación de todos los pacientes (100\%). El tiempo promedio de seguimiento fue $27,8 \pm 17,7$ meses (rango 6 - 72 meses), que correspondió a 530 años-paciente.

Durante el seguimiento fallecieron 17 pacientes $(7,7 \%)$ : 8 pacientes fallecieron por causa cardiovascular ( 3 por muerte súbita, 2 por Infarto agudo al miocardio, 2 por Insuficiencia cardíaca y 1 por endocarditis de prótesis) y 9 fallecen por otras causas (5 por cáncer, 3 por infecciones y uno por traumatismo de cráneo).

Se logró seguimiento clínico en 192 pacientes, que sobrevivieron a la cirugía $(89,6 \%)$. El seguimiento clínico promedio fue 7,48 $\pm 12,1$ meses (rango $1-64$ meses) y corresponde a 120 años-paciente.

Durante el seguimiento no se registraron eventos tromboembólicos ni hemorrágicos.

Discusión: El reemplazo valvular aórtico se ha convertido en una de las cirugías cardíacas más frecuentes actualmente en los países desarrollados, fenómeno que también hemos observado en nuestro centro. Durante el año 2012 realizamos 679 cirugías cardíacas, de las cuales $167(24,6 \%)$ correspondieron a reemplazos valvulares aórticos.

El año 2006 introdujimos el uso de bioprótesis en nuestro centro. Las primeras prótesis que implantamos correspondieron a las prótesis porcinas Carpentier Edwards ${ }^{\circledR}$ y posteriormente, el año 2009, a prótesis Biocor ${ }^{\circledR}$. Utilizamos ocasionalmente algunas prótesis Hancock II ${ }^{\circledR}$ y Perimount ${ }^{\circledR}$. Debido a que el manejo y el riesgo de complicaciones es el mismo, decidimos incluirlos a todos en esta serie.

Además, del aumento en el número de pacientes, hemos observado una tendencia al mayor uso de bioprótesis en 
relación a las prótesis mecánicas. Entre el 2006 y el 2011 realizamos 570 reemplazos valvulares aórticos y utilizamos bioprótesis en $286(50,2 \%)$. La proporción de bioprótesis ha ido en aumento cada año, llegando el 2012, a un $80,8 \%$ (135 de los 167 reemplazos valvulares aórticos realizados). Esta tendencia es similar a la observada en los registros europeos y norteamericanos, donde más del $70 \%$ de los implantes actuales corresponden a biopóte$\operatorname{sis}^{2,15}$.

La indicación de bioprótesis en nuestro hospital se hace en base a las recomendaciones internacionales, considerando la edad mayor de 60 años como el principal criterio en la indicación ${ }^{5}$. Sin embargo en nuestra población existen pacientes con poco acceso a centros de salud (ruralidad), que desempeñan actividades de riesgo (obreros de la construcción, obreros forestales, pescadores), mujeres en edad fértil, pacientes con dificultades para seguir un tratamiento anticoagulante (analfabetos, alcohólicos) y cada vez más jóvenes que prefieren asumir el riesgo de una reoperación en el futuro que la obligación de asumir un tratamiento anticoagulante. Esto explica por qué hubo 52 pacientes menores de 60 años en esta serie.

La mortalidad operatoria observada en el reemplazo valvular aórtico aislado fue 2,2\% y la mortalidad global fue de $3,5 \%$. Estos resultados son comparables a la mortalidad de $1,6 \%$ publicada por el grupo de la Universidad Católica para reemplazo valvular aórtico aislado $^{16}$, y a los publicados por el Instituto Nacional del Tórax y los registros europeos y norteamericanos que describen una mortalidad global para el reemplazo valvular aórtico entre 2,6 y 4,6\% ${ }^{17-19}$. El resto de las complicaciones observadas fue similar a lo publicado en otras series nacionales ${ }^{16,18,20 .}$

El uso de aspirina en reemplazo valvular aórtico con bioprótesis fue propuesto por varios autores en la década de 1990 y principios del 2000, demostrando la seguridad de reemplazar la anticoagulación formal por aspirina en el periodo postoperatorio inmediato ${ }^{11-15}$.

En nuestro hospital el TACO está centralizado en un policlínico a cargo de enfermeras que realizan los controles, existe un protocolo de manejo de las dosis y se realiza control por médico para los casos de difícil ajuste o niveles inadecuados. Se realizan en promedio 1650 controles mensuales. De acuerdo nuestros registros, sólo un 42,1\% de los pacientes se encuentra en niveles de INR óptimos al momento del control, un 34,7\% se encuentra bajo, y un $23,2 \%$ se encuentra por sobre el nivel deseado.
Considerando las dificultades que puede tener el tratamiento anticoagulante en nuestros pacientes, nuestro grupo adoptó el uso de aspirina desde el momento de la introducción de las bioprótesis en nuestro centro.

La tasa de complicaciones tromboembólicas observada en esta serie fue baja, con un 3,5\% de eventos embólicos antes de 30 días y ausencia de estas complicaciones en los dos meses posteriores. Esto es similar a las tasas de 2,6 a $3,7 \%$ descritas por otros autores ${ }^{12}-14$.

Nuestra serie es pequeña como para identificar factores asociados a estos eventos. Bardissi y cols, estudiaron 861 pacientes y compararon las complicaciones tromboembólicas utilizando warfarina versus aspirina. Observaron tasas de eventos tromboembólicos de 5\% a 90 días en ambos grupos (NS). Los factores de riesgo de eventos en esa serie fueron el género femenino, la capacidad funcional III / IV y el uso de prótesis de $19 \mathrm{~mm}^{21}$.

Brennan, utilizando los datos de la Sociedad de Cirujanos Torácicos de Estados Unidos, comparó el uso de aspirina sola, warfarina sola y aspirina más warfarina en reemplazo valvular aórtico con bioprótesis en mayores de 65 años (25.656 pacientes). No encontró diferencias entre el uso de aspirina versus warfarina exclusivas, y observó un beneficio en reducir el riesgo de mortalidad y embolías con el uso combinado de ambos medicamentos, asociado a una mayor tasa de sangrado ${ }^{22}$.

Otros autores como Brueck han ido más lejos y han publicado que no hay diferencias en las tasas de complicaciones entre aquellos en que se usa y los que no se usa aspirina, $\mathrm{y}$ han propuesto no usar ninguna terapia en estos pacientes $^{23}$.

El año 2006, cuando iniciamos esta serie, las recomendaciones y guías de manejo clínico recomendaban el uso de anticoagulación durante los primeros tres meses de la intervención. La evidencia actual ha permitido modificar esas recomendaciones. Las guías europeas actuales de manejo de patología valvular recomiendan el uso de aspirina exclusiva en reemplazo valvular aórtico con bioprótesis, confirmando que su uso es seguro para estos pacientes ${ }^{5}$.

Limitaciones: Por tratarse de un estudio retrospectivo, no se realizó una búsqueda dirigida de complicaciones y sólo se registran los eventos clínicos. Mediante el uso de tomografía computada y resonancia nuclear magnética se ha demostrado la presencia de microembolias en muchos de los pacientes sometidos a reemplazo valvular. Sin embargo, la importancia clínica de estos hallazgos es 
relativa y no siempre se acompañan de síntomas o déficits detectables. Debido a que no utilizamos rutinariamente estos exámenes, es probable que exista una tasa de complicaciones sub clínicas no detectadas.

Debido a que somos un centro de referencia que interviene pacientes que vienen de otros hospitales, algunos eventos ocurridos pudieron ser resueltos en sus hospitales de origen y no ser informados. El seguimiento con los datos del Registro Civil fue de $100 \%$, por lo que podemos asumir que las complicaciones no detectadas no fueron fatales.

\section{Conclusión}

El uso exclusivo de aspirina es seguro en prevenir eventos tromboembólicos en pacientes operados de reemplazo valvular aórtico con prótesis biológica.

\section{Referencias:}

1.- D'ARCY JL, PRENDERGAST BD, CHAMBERS JB, RAY SG, BRIDGEWATER B. Valvular heart disease: the next cardiac epidemic. Heart 2011; 97: 91-3.

2.- BRIDGEWATER B, KINSMAN R, WALTON P, GUMMERT J, KAPPETEIN AP. The 4th European Association for Cardio-Thoracic Surgery adult cardiac surgery database report. Interact Cardiovasc Thorac Surg 2011; 12: 4-5.

3.- EZEKOWITZ MD. Anticoagulation management of valve replacement patients. J Heart Valve Dis 2002; 11: S56-60.

4.- CANNEGIETER SC, ROSENDAAL FR, WINTZEN AR, VAN DER MEER FJ, VANDENBROUCKE JP, BRIËT E Optimal oral anticoagulant therapy in patients with mechanical heart valves. N Engl J Med 1995; 333:11-7.

5.- VAHANIAN A, ALFIERI O, ANDREOTTI F, ANTUNES MJ, BARÓN-ESQUIVIAS G, BAUMGARTNER H, et al. The Joint Task Force on the Management of Valvular Heart Disease of the European Society of Cardiology (ESC) and the European Association for Cardio-Thoracic Surgery (EACTS). The new ESC/EACTS guidelines on the management of valvular heart disease. Arch Cardiovasc Dis 2012; 105: 465 - 7 .
6.- GOHLKE-BÄRWOLF C, ACAR J, OAKLEY C,BUTCHART E, BURCKHART D, BODNAR E, et al. Guidelines for prevention of thromboembolic events in valvular heart disease. Study Group of the Working Group on Valvular Heart Disease of the European Society of Cardiology. Eur Heart J 1995; 16: 1320 - 30.

7.- BONOW RO, CARABELLO B, DE LEON AC, EDMUNDS LH JR, FEDDERLY BJ, FREED MD, et al. Committee on Management of Patients With Valvular Heart Disease. ACC/AHA Guidelines for the Management of Patients With Valvular Heart Disease. J Heart Valve Dis 1998; 7: 672-707.

8.- STEIN PD, ALPERT JS, BUSSEY HI, DALEN JE, TURPIE AG. Antithrombotic therapy in patients with mechanical and biological prosthetic heart valves. Chest 2001; 119: $220 \mathrm{~S}-227 \mathrm{~S}$.

9.- BUTCHART EG, GOHLKE-BÄRWOLF C, ANTUNES MJ, TORNOS P, DE CATERINA R, CORMIER B, et al; Working Groups on Valvular Heart Disease, Thrombosis, and Cardiac Rehabilitation and Exercise Physiology, European Society of Cardiology. Recommendations for the management of patients after heart valve surgery. Eur Heart J 2005; 26: 2463 - 71 . 
10.- DUNNING J, VERSTEEGH M, FABBRI A, PAVIE A, KOLH P, LOCKOWANDT U, et al. EACTS Audit and Guidelines Committee. Guideline on antiplatelet and anticoagulation management in cardiac surgery. Eur J Cardiothorac Surg 2008; 34: 73 - 92.

11.- BABIN-EBELL J, SCHMIDT W, EIGEL P, ELERT O. Aortic bioprosthesis without early anticoagulation--risk of thromboembolism. Thorac Cardiovasc Surg 1995; 43: $212-4$

12.- MOINUDDEEN K, QUIN J, SHAW R, DEWAR M, TELLIDES G, KOPF G, et al. Anticoagulation is unnecessary after biological aortic valve replacement. Circulation 1998; 98: II95-8.

13.- GHERLI T, COLLI A, FRAGNITO C, NICOLINI F, BORRELLO B, SACCANI S, et al. Comparing warfarin with aspirin after biological aortic valve replacement: a prospective study. Circulation 2004; 110: 496 - 500.

14.- SUNDT TM, ZEHR KJ, DEARANI JA, DALY RC, MULLANY CJ, MCGREGOR CG, et al. Is early anticoagulation with warfarin necessary after bioprosthetic aortic valve replacement? J Thorac Cardiovasc Surg 2005; 129: 1024 - 31.

15.- EL-HUSSEINY M, SALHIYYAH K, RAJA SG, DUNNING J. Should warfarin be routinely prescribed for the first three months after a bioprosthetic valve replacement? Interact Cardiovasc Thorac Surg 2006; 5: 616 - 23.

16.- BECKER P, RAMÍREZ A, CIFUENTES I, REBOLLEDO R, ZALAQUETT R, MORÁN S, et al. Morbimortalidad precoz y alejada del reemplazo valvular aórtico con prótesis mecánica y biológica durante la última década: El estándar de oro. Rev Med Chil 2009; 137: 1153 - 62.

17.- BROWN JM, O'BRIEN SM, WU C, SIKORA JA, GRIFFITH BP, GAMMIE JS, et al. Isolated aortic val- ve replacement in North America comprising 108,687 patients in 10 years: changes in risks, valve types, and outcomes in the Society of Thoracic Surgeons National Database. J Thorac Cardiovasc Surg 2009; 137: 82 - 90.

18.- VILLAVICENCIO M, TURNER E, NARANJO L. Resultados del reemplazo valvular aórtico con prótesis mecánica en el Instituto Nacional del Tórax. Rev Med Chil 2005; 133: $1161-72$.

19.- The Society of Thoracic Surgeons. Adult cardiac surgery database, executive summary, 10 years STS report.

http://www.sts.org/sites/default/files/documents/20112ndHarv estExecutiveSummary.pdf

20.- ZALAQUETT R, OLIVARES G, MEJÍA R, QUEZADA F, PADILLA O, BECKER P, et al. Reemplazo valvular aórtico con prótesis biológica en pacientes mayores de 70 años con estenosis aórtica. Rev Med Chil 2011; 139: 150-157.

21.- ELBARDISSI AW, DIBARDINO DJ, CHEN FY, YAMASHITA MH, COHN LH. Is early antithrombotic therapy necessary in patients with bioprosthetic aortic valves in normal sinus rhythm? J Thorac Cardiovasc Surg 2010; 139: $1137-45$.

22.- BRENNAN JM, EDWARDS FH, ZHAO Y, O'BRIEN S, BOOTH ME, DOKHOLYAN RS, et al; DEcIDE AVR Research Team. Early anticoagulation of bioprosthetic aortic valves in older patients: results from the Society of Thoracic Surgeons Adult Cardiac Surgery National Database. J Am Coll Cardiol 2012; 60: 971 - 7.

23.- BRUECK M, KRAMER W, VOGT P, STEINERT N, ROTH P, GÖRLACH G, ET AL. Antiplatelet therapy early after bioprosthetic aortic valve replacement is unnecessary in patients without thromboembolic risk factors. Eur J Cardiothorac Surg 2007; 32: 108 - 12. 